\title{
Desenvolvimento de um programa computacional para implantação econômica de lavouras canavieiras
}

\author{
Tadeu A. Marques', Gil E. Serra² \& Patrícia A. A. Marques ${ }^{3}$
}

\begin{abstract}
RESUMO
Desenvolveu-se um algoritmo para a implantação convencional de lavouras canavieiras e em adição, uma implantação diferenciada com a finalidade de proporcionar produções, em toneladas, iguais nos anos subseqüentes à implantação, visto que, com o passar dos anos, as produtividades da cana-de-açúcar são diferentes; contudo, a nova metodologia fica limitada à área máxima de plantio, sendo que, em alguns casos, a produção anual cai, devido à impossibilidade de aumento da área; ampliou-se então, uma terceira metodologia, na qual a área não se torna fator limitante e, portanto, as produções se mantêm constantes. Para avaliar a viabilidade dos métodos propostos, os cálculos dos custos de implantação da cultura, da manutenção da soqueira, de renda bruta anual e das rendas líquidas, foram trazidas ao Valor Presente. Os resultados apontam que, apesar do maior investimento inicial, os métodos inovadores sempre proporcionam maiores lucratividades reais com o passar do tempo e, ainda, que o tempo de recuperação do capital investido também é menor. Os algoritmos foram transformados no software "canavial.exe", o qual pode ser obtido gratuitamente no endereço da Internet: "http://dl.winsite.com/bin/downl?500000027058".
\end{abstract}

Palavras-chave: cana-de-açúcar, modelo computacional, dimensionamento, instalação

\section{Development of a computer program for economical implantation of sugarcane plantations}

\begin{abstract}
An algorithm was developed for conventional implantation of sugarcane crap. In addition, a differentiated implantation was developed with the purpose of providing same production (tons), in subsequent years of the implantation. Since sugarcane productivities in the following years are different. However the new methodology is limited to the maximum planting area, and in some cases annual production falls due to impossibility to increase planted area. A third methodology was also developed, in which the area does not become a limiting factor, and therefore production remain constant. To evaluate the viability of proposed methods, calculations of the cost of crop implantation, of sugarcane maintenance, annual income and the net incomes were converted later to the Present Value. The results showed that in spite of the higher initial investment, the new methods always provide highest real profit abilities in the time course and that recovery time of the invested capital is also smaller. The algorithms were transformed in the software "canavial.exe", which can be assessed freely at site: "http://dl.winsite.com/bin/downl?500000027058".
\end{abstract}

Key words: sugar-cane, computer model, installation

${ }^{1}$ CCA/UNIOESTE - Campus II, Rodovia Raposo Tavares, km 572 - Bairro Limoeiro, CEP 19067-175 Presidente Prudente, SP . Fone (0xx18) 229-2000 E-mail: tmarques@prudenet.com.br

2 UNICAMP, Cidade Universitária Zeferino Vaz, Barão Geraldo, CEP 13083-970, Campinas, SP, Fone(19) 3835-5758 E-mail: gilserra@terra.com.br ${ }^{3}$ ESALQ/USP, Av. Pádua Dias, 11. CP 9. CEP 13418-900 Piracicaba, SP. Fone (018) 231-3474. E-mail: pmarques@prudenet.com.br 


\section{INTRODUÇÃO}

A colheita da cana-de-açúcar (Saccharum spp.), cultura semiperene, pode ser realizada doze meses após o plantio (sistema denominado cana de ano) ou dezoito meses depois (cana de ano e meio); posterior à colheita inicial, pode-se realizar cortes consecutivos, denominados corte das canas socas (Fernandes, 2000); contudo, um grande problema enfrentado pela maioria dos técnicos das empresas canavieiras é a oscilação da matéria-prima produzida, com o passar dos anos (Brugnaro, 1983). Com o decorrer dos anos e dependendo dos tratos culturais, do controle do tráfego e conseqüente compactação do solo, a produtividade da cana-deaçúcar diminui em proporções diferentes, sendo fatores interferentes o tipo de solo, as condições climáticas e as cultivares utilizadas, entre outros (Matsuoka et al., 1995). A determinação do valor real do custo de produção da cana é diferente para cana de ano e cana de ano e meio, pois o tempo de espera para a colheita, ou seja, o número de períodos envolvidos para se calcular o Valor Presente (VP) desta colheita, também é diferente; no entanto é recomendável plantar cana de ano e meio, de vez que esta fica mais tempo no campo, vegeta mais e, conseqüentemente, proporciona melhores produções em toneladas (Matsuoka, 1996). Segundo Stupiello (1989) um bom planejamento agrícola contribui na setorização da lavoura e conseqüente distribuição de cultivares. Conforme Brugnaro (1983) a implantação de um canavial deve ser bem realizada, visto poder ficar durante três a quatro safras ou mais e, se implantada de modo incorreto, poderá proporcionar prejuízos; o autor apresenta três métodos de implantação de canavial: 1) áreas anuais com plantios constantes; 2) um ano com área dobrada e 3) dois anos com área dobrada, sendo que utilizou, para o modelo, cana-deaçúcar em áreas com quatro cortes. Calliari (2001), cita que existem localidades onde a cana-de-açúcar proporciona até 10 cortes em uma mesma área, fato que agrava ainda mais a incorreta implantação do canavial. Fernandes (2000) apresenta um modelo matemático para simulação do custo de produção da cana-de-açúcar, no qual salienta a importância de se colocar as despesas e receitas corrigidas ao "valor presente" e que, para tanto, se deve eleger uma taxa para remuneração do capital.

Weick (2001), estudando as mudanças da tecnologia do “agribusiness" para 2010, cita que grandes inovações mudarão o panorama internacional e, dentre elas, que quatro tecnologias serão as mais importantes, sendo: 1) Sistema de Posicionamento Global (GPS), 2) Sistema de Informação Geográfica (SIG), 3) Biotecnologia e 4) Informática aliada à Internet. Ascoug et al. (1999) em uma análise do uso do computador por agricultores norte-americanos, afirmam que a adoção do computador nas propriedades agrícolas depende de alguns fatores, a saber: nível de escolaridade do produtor, idade do produtor, tamanho da propriedade, preço do produto agrícola produzido e diversificação das atividades, sendo que em grandes propriedades mais de $86 \%$ utilizam computador e, ainda, que entre os pequenos produtores este número se reduz drasticamente, contudo, a maioria dos produtos agrícolas comercializados vem de propriedades que utilizam recursos computacionais. Os mesmos autores relatam que aqueles que adotam o uso de computadores solicitam o desenvolvimento de programas que auxiliem na tomada de decisão, para que a possibilidade de erros seja minimizada. Antunes \& Angel (1996) afirmam que os recursos computacionais devem ser encarados pelos agricultores como fator de produção adicional e que devem ser adotados caso tragam benefícios financeiros compensadores. Dentro deste panorama procurou-se desenvolver um programa para a otimização da implantação de canaviais, de modo racional e econômico, sem se esquecer de levar em consideração a necessidade de fornecimento contínuo e homogêneo, da matéria-prima para a indústria e, também, a grande diversidade de produtividade nos cortes, subseqüentes para cultivares diferentes e variados números de cortes em áreas e situações diferentes.

\section{MATERIAL E MÉTODOS}

Para o desenvolvimento deste trabalho foram utilizados o sistema operacional Microsoft Windows 95 e o microcomputador PC Pentium II. Utilizou-se, também, o Programa Borland Delphi, para desenvolver a programação lógica e, para o dimensionamento da área a ser plantada anualmente usaram-se três metodologias:

a) Convencional, ou seja, com plantios anuais semelhantes (Eq. 1):

$$
\text { Área }_{(\mathrm{i}-1)}=\frac{\text { Área }_{\text {disp. }}}{\mathrm{NC}+\mathrm{k}} \quad \text { para } \mathrm{i}=1,2, \ldots, \mathrm{PL}
$$

sendo:

Área $(\mathrm{i}-1)$ - área de plantio para o ano (i-1), em ha

NC - número máximo de cortes permitido para a área e para esta cultivar

Área $_{\text {disp. }}$ - área total da lavoura canavieira para as condições estudadas

PL - número de anos de plantios

$\mathrm{k}$ - 0 para cana de ano e 1 para cana de ano e meio

Sujeita à seguinte restrição: se (i-1) $\geq \mathrm{NC} \rightarrow$ Reforma

b) Proposta, através da qual se tenta produzir ano a ano, independente das áreas plantadas mas se fixando a área to-

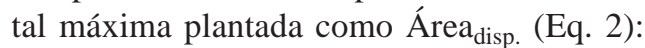

$$
\text { Área }_{(i-1)}=\frac{\frac{\sum_{j=1}^{N C} P_{j}}{N C} \cdot \text { Área }_{\text {disp. }}}{P_{j}} \quad \text { para } i=1,2, \ldots, P L
$$

sendo:

Área $(i-1)-$ área a ser plantada no ano (i-1), em ha

$\mathrm{P}_{\mathrm{J}}$ - produtividade no ano j, em toneladas de cana por ha

NC - número máximo de cortes permitido para a área e para esta cultivar

Área disp $_{-}$área total da lavoura canavieira para as condições estudadas

PL - número de anos de plantio 
E sujeita às seguintes restrições:

1. Se

$\sum_{(\mathrm{j}=1)}^{(\mathrm{i}-1)}$ Área $_{(\mathrm{i}-1)}>$ Área disp. $\rightarrow\left[\right.$ Área $_{(\mathrm{i}-1)}=$ Área disp . $-\sum_{(\mathrm{j}=1)}^{(\mathrm{i}-2)}$ Área $\left._{(\mathrm{i}-1)}\right]$

sendo que, após alguns anos e devido à área disponível, a produção irá cair um pouco.

2. Se (i-1) $\geq \mathrm{NC} \rightarrow$ Reforma

3. Se $\mathrm{i}>\mathrm{NC} \rightarrow \mathrm{j}=1$;

c) Proposta modificada, com a qual se tenta produzir constantemente, ano a ano, independente das áreas plantadas, não respeitando a área máxima total plantada como ÁREA disp, (Eq. 3):

$$
\text { Área }_{(i-1)}=\frac{\frac{\sum_{j=1}^{N C} P_{j}}{N C} \cdot \text { Área }_{\text {disp. }}}{P_{j}} \quad \text { para } \mathrm{i}=1,2, \ldots, P L
$$

no qual:

Área ${ }_{(\mathrm{i}-1)}$ - área a ser plantada no ano (i-1), em ha

$\mathrm{P}_{\mathrm{J}}$ - produtividade no ano $\mathrm{j}$, em toneladas de cana por ha

NC - número máximo de cortes permitido para a área e para esta cultivar

Área $_{\text {disp. }}$ - área total da lavoura canavieira para as condições estudadas

PL - número de anos de plantio

E sujeita às seguintes restrições:

1. Se (i-1) $\geq \mathrm{NC} \rightarrow$ Reforma

2. Se $\mathrm{i}>\mathrm{NC} \rightarrow \mathrm{j}=1$;

As produções serão mantidas constantes, pois não existe restrição relativa ao tamanho da área.

Para o cálculo de custo, tem-se:

a) Custo de implantação de canavial (Eq. 4):

$$
\mathrm{C}_{\mathrm{i}}=\sum_{\mathrm{j}=0}^{\mathrm{i}}\left(\mathrm{AP}_{\mathrm{j}}\right) \cdot \text { Valor, } \quad \text { para } \mathrm{i}=0,1,2, \ldots, \mathrm{PL}
$$

donde:

$\mathrm{C}_{\mathrm{i}}$ - custo com implantação de canavial no ano i, em dólares

$\mathrm{AP}_{\mathrm{J}}$ - área de plantio no ano j, em ha

PL - número de anos de plantio

Valor - gasto para plantio de um hectare, em dólar

b) Custo dos tratos com soca, inclusive colheita (Eq. 5):

$$
\mathrm{CS}_{\mathrm{i}}=\sum_{\mathrm{j}=2}^{(\mathrm{i}-1)}\left(\mathrm{PST}_{\mathrm{j}} \cdot \mathrm{VST}\right), \quad \text { para } \mathrm{i}=1,2, \ldots, \mathrm{PL}
$$

no qual:

$\mathrm{CS}_{\mathrm{i}}$ - custo com tratos nas soqueiras no ano i, em dólar

$\mathrm{PST}_{\mathrm{j}}$ - produção de soca em toneladas, para o ano j

VST - gasto com a soca por toneladas esperadas, em dólar

PL - número de anos de plantio c) Renda Bruta (Eq. 6):

$\mathrm{RB}_{\mathrm{i}}=\left[\sum_{\mathrm{j}=1}^{\mathrm{i}}\left(\mathrm{PCPT}_{\mathrm{j}}\right)+\sum_{\mathrm{j}=2}^{\mathrm{i}}\left(\mathrm{PST}_{\mathrm{j}}\right)\right] \cdot$ Pr eço, $\quad$ para $\mathrm{i}=1,2, \ldots, \mathrm{PL}$

donde:

$\mathrm{RB}_{\mathrm{i}}$ - renda bruta no ano i, em dólar

$\mathrm{PCPT}_{\mathrm{j}}$ - produção de cana planta no ano $\mathrm{j}$, em toneladas

$\mathrm{PST}_{\mathrm{j}}$ - produção de soca em toneladas, para o ano j

Preço - preço básico da tonelada de cana, em dólar

PL - número de anos de plantio

d) Renda líquida (Eq. 7):

$$
\mathrm{RL}_{\mathrm{i}}=\left[\mathrm{RB}_{\mathrm{i}}-\mathrm{CS}_{\mathrm{i}}-\mathrm{C}_{\mathrm{i}}\right] \quad \text { para } \mathrm{i}=1,2, \ldots, \mathrm{PL}
$$

sendo: $\mathrm{RL}_{\mathrm{i}}$ - renda líquida do ano $\mathrm{i}$, em dólar

e) Renda líquida acumulada (Eq. 8):

$$
\mathrm{RLA}_{\mathrm{i}}=\sum_{\mathrm{i}=1}^{\mathrm{i}} \mathrm{RL}_{\mathrm{i}} \quad \text { para } \mathrm{i}=1,2, \ldots, \mathrm{PL}
$$

na qual:

$\mathrm{RLA}_{\mathrm{i}}$ - renda líquida acumulada no ano i, em dólar

f) Renda líquida corrigida para valor presente (Eq. 9):

$$
\operatorname{RLC}_{i}=\frac{\mathrm{RL}_{\mathrm{i}}}{\left(1+\frac{\operatorname{taxa}}{100}\right)^{\mathrm{i} \cdot \mathrm{j}}} \quad \text { para } \mathrm{i}=1,2, \ldots, \mathrm{PL}
$$

sendo:

$\mathrm{RLC}_{\mathrm{i}}$ - renda líquida corrigida para valor presente, do ano i, em dólar (Frizzone et al., 1994)

Taxa - taxa de (juros + correção) mensal, em dólar e em porcentagem

j - número de meses para cana de ano e meio, no primeiro corte é igual a 18; para os demais casos, igual a 12

g) Renda líquida corrigida acumulada (Eq. 10):

$$
\operatorname{RLCA}_{\mathrm{i}}=\sum_{\mathrm{i}=1}^{\mathrm{i}} \mathrm{RLC}_{\mathrm{i}} \quad \text { para } \mathrm{i}=1,2, \ldots, \mathrm{PL}
$$

sendo: $\mathrm{RLCA}_{\mathrm{i}}$ - renda líquida corrigida acumulada no ano i, em dólar

\section{RESULTADOS E DISCUSSÃO}

O presente trabalho resultou na elaboração do programa "canavial.exe", que pode ser obtido no endereço da Internet: “http://dl.winsite.com/bin/downl?500000027058”. O mesmo apresenta a tela de entrada (Figura 1), com os dados inicializados, segundo Agrianual (2001), mas que podem ser alterados de acordo com a conveniência do usuário. É importante lembrar que esses valores devem ser preenchidos em dólar.

Preenchendo-se os valores adequadamente, poder-se-á escolher simulação para cana de ano ou para cana de ano e 


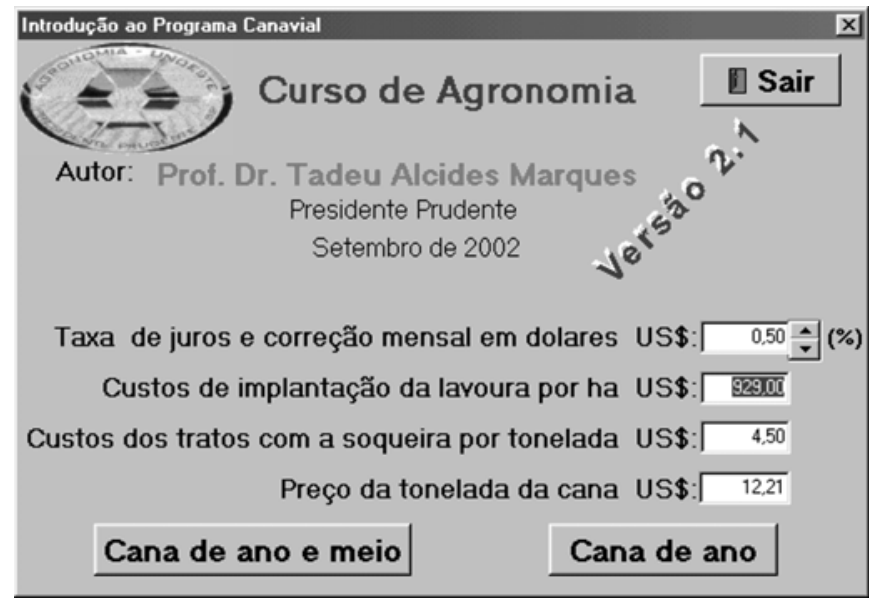

Figura 1. Tela inicial do programa canavial.exe

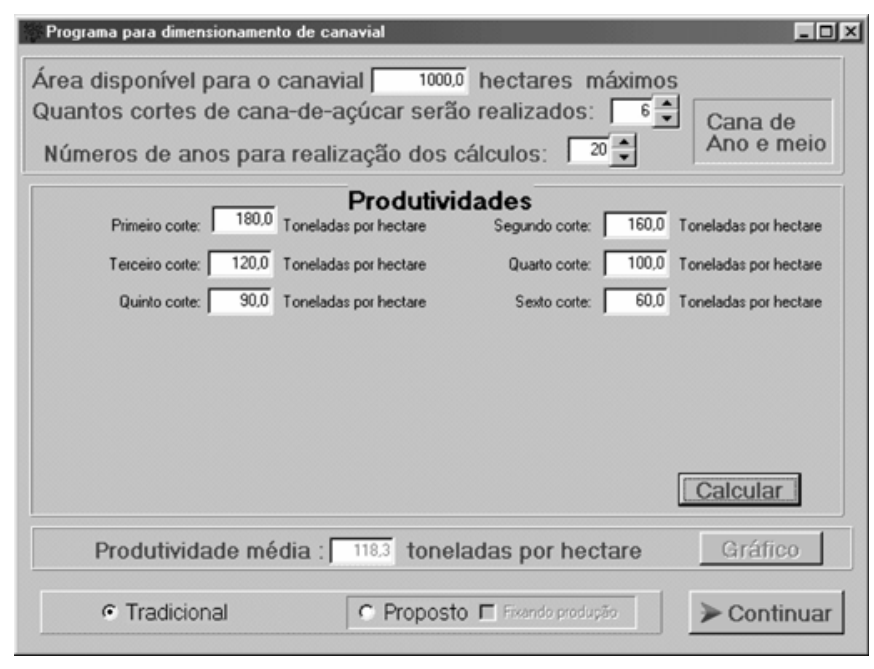

Figura 2. Para escolha da área, número de cortes, número de anos para realização dos cálculos e produtividades anuais

meio, sendo que, ao apertar o botão equivalente, aparecerá a tela de seqüência (Figura 2).

Na tela da Figura 2 deve-se apertar o botão calcular, o qual procede ao cálculo da produtividade média geral, de acordo com os valores digitados. O programa permite fazer-se a simulação para no máximo 15 cortes e para 100 anos de cálculo.

Depois de escolhidas as opções e apertado o botão calcular, deve-se escolher uma das opções: tradicional (método convencional de dimensionamento) ou proposto (método proposto para dimensionamento da lavoura canavieira), que apresenta a possibilidade de não fixar a produção, caso em que se respeita a área máxima disponível para o plantio, ou se fixa a produção, mantendo-a constante todos os anos, podendo passar um pouco da área máxima disponível, sendo esta a única diferença entre o método proposto e o proposto modificado. Após esta seleção, apertar o botão continuar, o qual levará para o próximo formulário (Figura 3).

A Figura 3 mostra uma visão parcial da planilha de resultados para o dimensionamento tradicional, para cana de ano e meio, com o total de produção em 20 anos e produção média anual; com as barras de rolagem, pode-se ver o res- tante das colunas e linhas. Nesta “Janela” existe o botão "voltar" que, se selecionado, voltará para a tela anterior (Figura 2). Se o outro método de cálculo for eleito e se pressionar “continuar”, aparecerá uma tela semelhante, apenas com outros dados, pois os cálculos serão executados com o "modelo proposto” ou "proposto fixando a produção”. Ao se voltar novamente para a tela da Figura 2, observar-se-á que o botão gráfico está liberado e, se for selecionado, apresentará a próxima tela (Figura 4).

Na Figura 4, observa-se o gráfico inicial das produções anuais comparativas para o método convencional e o método proposto, onde podem ser observadas as maiores produções do método proposto em relação ao método convencional, nos anos iniciais da exploração deste canavial. Este gráfico pode ser impresso e, com o botão da direita do mouse sobre o gráfico, pode-se escolher salvá-lo ou copiá-lo para a área de transferência para, posteriormente, colá-lo em outro aplicativo. Observam-se mais dois botões "lucros reais anuais" e "lucros acumulados reais” que, quando selecionados, mostrarão as

\begin{tabular}{|c|c|c|c|c|c|c|c|c|}
\hline \multicolumn{8}{|c|}{ Cálculos para dimensionamento de canavial tradicional [cana de ano e meio] } & Elax \\
\hline ANO & Plarkar (ha) & Colheta (ha) & Produczofition) & 1 Conte (ha) & 2 Conte (has) & 3 Corte (has & 4 Conte (has) & 5 cort $A$ \\
\hline 0 & 142.9 & & & & & & & \\
\hline 1 & 142.9 & 142.9 & 25714.3 & 142.9 & 0 & 0 & 0 & 0 \\
\hline 2 & 142.9 & 285,8 & 48571,4 & 142.9 & 142.9 & 0 & 0 & 0 \\
\hline 3 & 142,9 & 428.7 & 65714.3 & 142.9 & 142.9 & 142.9 & 0 & 0 \\
\hline 4 & 142,9 & 571.6 & 80000,0 & 142.9 & 142,9 & 142.9 & 142.9 & 0 \\
\hline 5 & 142.9 & 714.5 & 92857.1 & 142,9 & 142.9 & 142.9 & 142,9 & 142.9 \\
\hline 6 & 142.9 & 857.4 & 101428.6 & 142,9 & 142.9 & 142.9 & 142.9 & 142.9 \\
\hline 7 Reforms & 142,9 & 857.4 & 101428.6 & 142,9 & 142,9 & 142.9 & 142.9 & 142,9 \\
\hline 8Reforma & 142,9 & 857.4 & 101428.6 & 142,9 & 142.9 & 142,9 & 142.9 & 142,9 \\
\hline 9Reformo & 142.9 & 857.4 & 101428.6 & 142.9 & 142.9 & 142,9 & 142,9 & 142,9 \\
\hline 10 Reforma & 142.9 & 857.4 & 101428,6 & 142,9 & 142,9 & 142,9 & 142,9 & 142,9 \\
\hline 11 Reforms & 142,9 & 857.4 & 101428.6 & 142,9 & 142,9 & 142,9 & 142,9 & 142,9 \\
\hline$a^{n-1}$ & $m n$ & neר. & antemer & $m n$ & $m n$ & $m n$ & $m n$ & 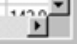 \\
\hline & ollar & & & crão total de 1 & $\begin{array}{l}1834286,1 \text { ton } \\
91714,3 \text { ton }\end{array}$ & neladas em 20 & DRetorma anc & \\
\hline
\end{tabular}

Figura 3. Apresentação da planilha de plantio, custos, renda líquida e renda líquida corrigida para cada ano

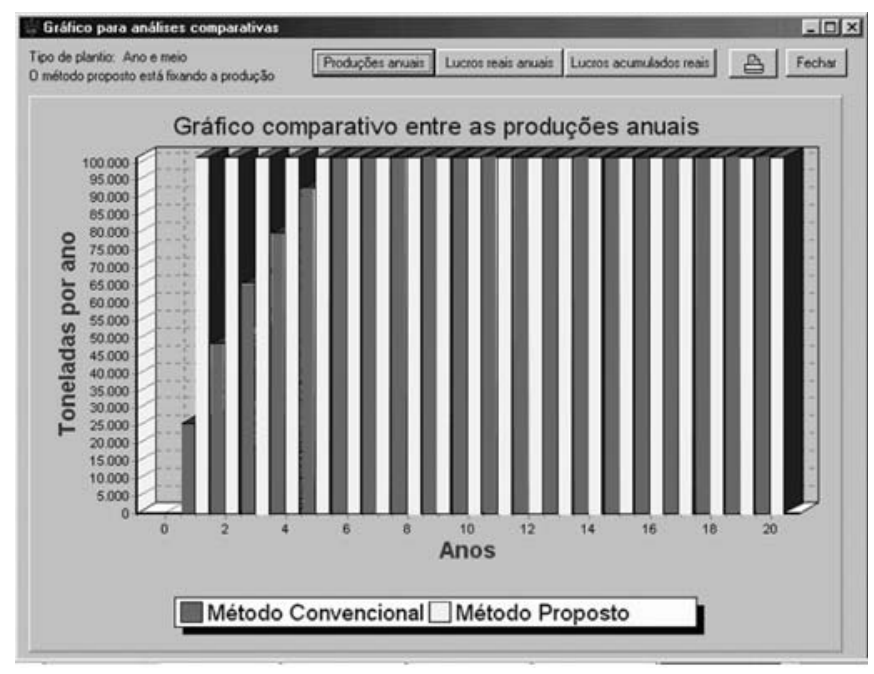

$\overline{\text { Figura 4. Análise comparativa entre o método convencional e o proposto }}$ 


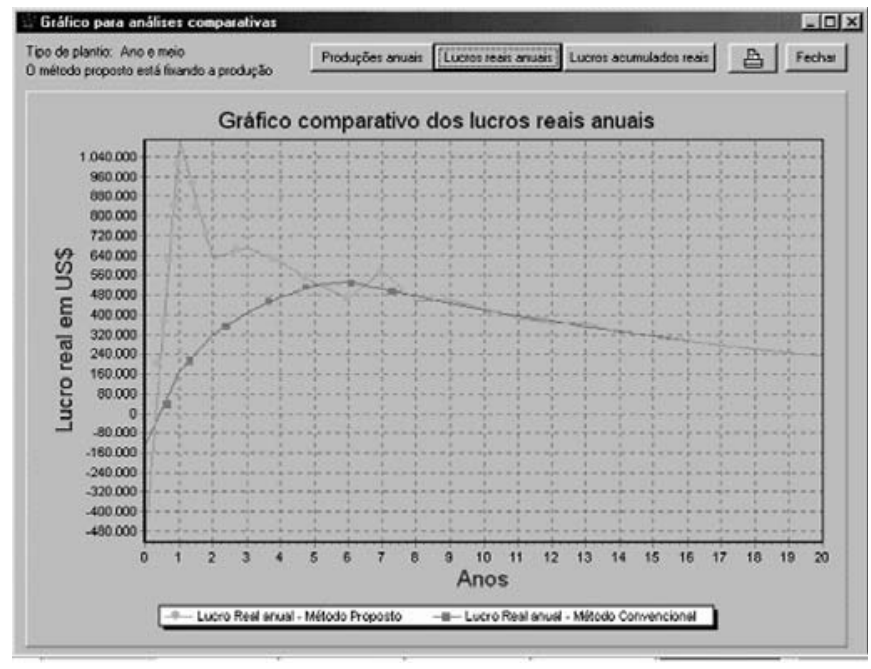

Figura 5. Análise comparativa dos lucros reais anuais para os métodos proposto e convencional

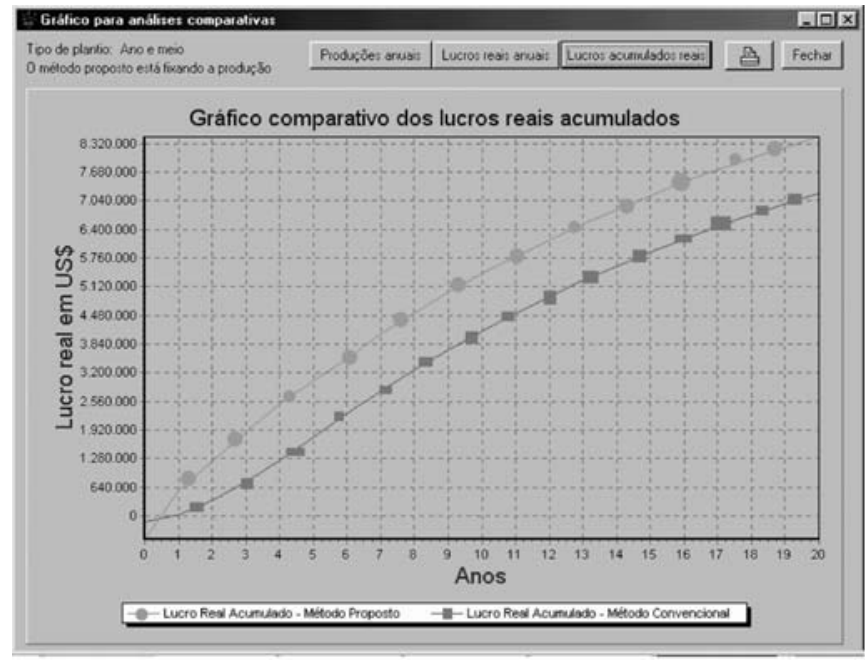

Figura 6. Análise comparativa dos lucros reais acumulados para os métodos proposto e convencional

telas referentes à Figura 5 e à Figura 6, respectivamente, as quais podem ser impressas, salvadas e copiadas.

Na Figura 5, a área abaixo das curvas apresenta o lucro total obtido, sendo que o método proposto mostra valores de lucros mais elevados nos anos iniciais e essas diferenças tendem a valor zero com, neste exemplo, em cinco a sete anos, período coincidente com o tempo de exploração médio de um canavial.

Na Figura 6 pode-se observar que o método proposto parte de valores mais negativos, visto a necessidade de maiores investimentos iniciais; contudo, esses investimentos adicionais são recuperados no primeiro ano de colheita e, posteriormente, o diferencial obtido em favor do método proposto mantêm-se pela vida do canavial.

\section{CONCLUSÕES}

1. Com o presente programa pode-se simular diferentes tipos de plantio para diferentes cultivares de cana-de-açúcar e se pode optar plantio tradicional ou, ainda, pelo plantio proposto, de acordo com o resultado da renda líquida real obtida no programa.

2. O programa pode ser ampliado e englobar um banco de dados com as unidades agrícolas de uma usina, setores, talhões e cultivares plantados para otimizar a lucratividade e manter um fornecimento de matéria-prima homogêneo para a indústria; pode-se, também, acrescentar ao programa dados relativos à produção de Açúcares Teoricamente Recuperáveis (ATR) para cada corte e o preço do ATR, otimizando e homogeneizando sua produção.

3. A única diferença observada entre o método proposto e o proposto modificado, é que o proposto modificado mantém a produção igual por todos os anos, sendo que em alguns deles a área máxima cultivada será maior que a área para cultivo necessitando, portanto, de incrementos nesta área.

\section{LITERATURA CITADA}

Agrianual. Anuário estatístico da agricultura brasileira. Para aumentar o lucro na cana-de-açúcar. São Paulo: FNP/M\&S, 2000. 2001 p.248-265

Antunes, L. M.; Angel, A. A informática na agropecuária. Guaíba: Agropecuária, 1996. 175p.

Ascough, J. C.; Hoag, D. L.; Frasier, W. M.; Mcmaster, G. S. Computer use in agriculture: an analysis of great plains producers. Computers and Electronics in Agriculture, Davis, v.2, p.189-204, 1999.

Brugnaro, C. Modelos aritméticos de implantação de canaviais. Cadernos Planalsucar, Piracicaba, v.2, n.1, p.3-10, 1983.

Calliari, M. Gerenciamento do canavial para otimização da produção de sacarose em usina sucroalcooleira.Campinas: FEAGRI/UNICAMP, 2001. 201p. Tese Doutorado

Fernandes, A. C. Cálculos na agroindústria da cana-de-açúcar. Piracicaba: STAB, 2000. 193p.

Frizzone, J. A.; Botrel, T. A.; Freitas, H. A. C. Análise comparativa dos custos de irrigação por pivô-central, em culturas de feijão, utilizando energia elétrica e óleo diesel. Engenharia Rural, Piracicaba, v.5, n.1, p.34-53, 1994.

Matsuoka, S. Botânica da cana-de-açúcar. In: Matsuoka, S. Botânica e ecofisiologia da cana-de-açúcar. Araras: UFSCar, 1996. cap I, p.1-9.

Matsuoka, S.; Arizono, H.; Bassinello, A. I.; Gheller, A. C. A.; Hoffmann, H. P.; Matsuda, Y. Variedades superprecoces de cana-deaçúcar. Álcool \& Açúcar, São Paulo, v.15, n.78, p.22-29, 1995.

Stupiello, J. P. Alguns aspectos de qualidade da matéria-prima. STAB-Açúcar, Álcool e Subprodutos, Piracicaba, v.7, n.3/5, p.52-54, 1989.

Weick, C. W. Agribusiness technology in 2010: Directions and challenges. Technology in Society, New York, v.23, p.59-72, 2001. 